\title{
Melhoria de Processos no Desenvolvimento de Software e Hardware - O Caso Maxtrack
}

\author{
Etiene Guerra ${ }^{1}$, Gustavo Horta Travassos ${ }^{1}$, \\ Gleison Santos ${ }^{2}$, Sômulo Mafra ${ }^{2}$, Ahilton Barreto ${ }^{2}$, Ana Regina Rocha ${ }^{2}$ \\ ${ }^{1}$ Maxtrack Industrial Ltda. \\ Av. Amazonas, 5416 - Nova Suiça - CEP: 30.480-000 - Belo Horizonte, MG \\ ${ }^{2}$ COPPE/UFRJ - Universidade Federal do Rio de Janeiro \\ Caixa Postal 68511 - CEP 21945-970 - Rio de Janeiro, RJ \\ \{eguerra, ght\} @maxtrack.com.br, \\ \{gleison, somulo, ahilton, darocha\}@cos.ufrj.br
}

\begin{abstract}
Maxtrack is a Brazilian company that produces logistic applications and automatic vehicle location solutions through the development and integration of both hardware and software. This paper describes Maxtrack experience in the deployment of a quality program aiming to improve the development of their software and hardware products. In January 2006 the company was successfully evaluated as CMMI Level 2. Difficulties, successes factors and lessons learned of the software process deployment program are also described in this paper.
\end{abstract}

Resumo. A Maxtrack é uma empresa brasileira, do segmento de rastreamento e logística, onde atua desenvolvendo, produzindo e integrando soluções de software e de hardware. Este artigo descreve a experiência da Maxtrack na implantação de um programa de qualidade objetivando melhorar o desenvolvimento do software e do hardware que compõem suas linhas de produtos. Este processo culminou em janeiro de 2006 com a obtenção do CMMI Nivel 2. São apresentadas as dificuldades encontradas, fatores de sucesso e lições aprendidas como resultado da implantação dos processos.

\section{Introdução}

A Maxtrack é uma empresa brasileira com mais de 06 anos de experiência no segmento de rastreamento e logística, onde atua desenvolvendo, produzindo e integrando soluções de software e de hardware. A empresa possui duas fábricas próprias, uma no Brasil e outra em Taiwan, com certificação ISO 9001 (ISO, 2001) no sistema de gestão da qualidade e ISO 14001 (ISO, 2004) no sistema de gestão ambiental nas atividades de "Fabricação, montagem, comercialização e assistência técnica de equipamentos eletrônicos". A Maxtrack tem como linha básica de atuação o desenvolvimento de soluções e a produção de equipamentos. O sistema desenvolvido pela Maxtrack torna possível o rastreamento e monitoramento de frotas em tempo real, podendo ser utilizado em aplicações de telemetria e em veículos particulares, indo desde operações simples até complexos sistemas logísticos.

Nos últimos anos, a Maxtrack apresentou um grande crescimento de participação no mercado nacional. Atualmente, o atendimento a tal mercado é realizado 
por uma rede de prestadores de serviços formada por mais de 40 distribuidores com abrangência em todo território nacional. O sucesso obtido abriu as portas do mercado internacional, principalmente na América do Sul e na Ásia. Em vista do crescimento apresentado, em 2004 foi iniciado na Maxtrack um programa de qualidade cujo principal objetivo era a melhoria na qualidade dos produtos de hardware e software desenvolvidos. Este programa foi interrompido temporariamente, sendo retomado no $2^{\circ}$ semestre de 2005.

Em uma decisão estratégica, a Alta Gerência da empresa optou pela adoção de processos aderentes ao Nível 2 do CMMI (Chrissis et al., 2003). Como principais justificativas para a adoção do modelo CMMI, podem ser citadas: (i) Necessidade de manter o desenvolvimento sob controle, pois o crescimento apresentado representou um aumento significativo no quadro de funcionários e na quantidade de projetos executados em paralelo; (ii) Aumentar a visibilidade da capacidade da empresa em desenvolver produtos com alta qualidade, pois a utilização de processos aderentes a um modelo reconhecido internacionalmente seria fundamental para a atuação no exterior; (iii) Necessidade de desenvolvimento cooperativo com parceiros internacionais, pois a Maxtrack possui entre seus parceiros internacionais organizações com avaliação CMMI no Nível 5, que exigem documentação de alta qualidade.

Como resultado da implantação desse programa de qualidade, a Maxtrack teve uma avaliação SCAMPI, no início de 2006, quando foi constatada a sua aderência ao Nível 2 do CMMI em sua representação em estágios. Este artigo descreve o caminho percorrido pela Maxtrack do início da implantação de seu programa de qualidade até a avaliação SCAMPI para o Nível 2 do CMMI. O texto está organizado em quatro seções, incluindo esta que introduz o artigo. $\mathrm{Na}$ seção 2, o foco é a implantação do programa visando à melhoria da qualidade no desenvolvimento e à avaliação CMMI Nível 2. $\mathrm{Na}$ seção 4 são apresentados os principais resultados e lições aprendidas. Por fim, na seção 5 , são apresentadas as conclusões e perspectivas futuras.

\section{A Busca pela Melhoria da Qualidade no Contexto da Maxtrack}

Os produtos desenvolvidos pela Maxtrack são caracterizados pela alta tecnologia empregada e envolvem, em sua maioria, a construção de hardware com software embarcado. Também há a criação de software específico para controle e gerenciamento dos dados manipulados pelos produtos de hardware e software, porém em menor escala. Outra característica da Maxtrack é o ciclo de desenvolvimento curto, devido à necessidade de rapidez na entrega dos produtos e de início da produção em larga escala. Essa rapidez na entrega é facilitada pelo fato da equipe de desenvolvimento ser bastante especializada e pela existência de famílias de produtos com características similares.

O desenvolvimento é considerado interno mesmo quando originado de uma necessidade específica de um cliente. $O$ produto a ser desenvolvido deve poder ser utilizado por clientes com diferentes características e objetivos, de forma a aumentar o poder de penetração no mercado. Dessa forma, a figura do "cliente" na empresa é representada pela Alta Gerência, que atua como identificador e avaliador de requisitos.

O principal nicho de mercado da Maxtrack é o desenvolvimento de soluções integradas de hardware e software. Neste caso, o produto possui um ciclo de vida semelhante ao apresentado na Figura 1. Como pode ser percebido nos itens em cinza, o processo de desenvolvimento ocupa apenas uma parte deste fluxo. 


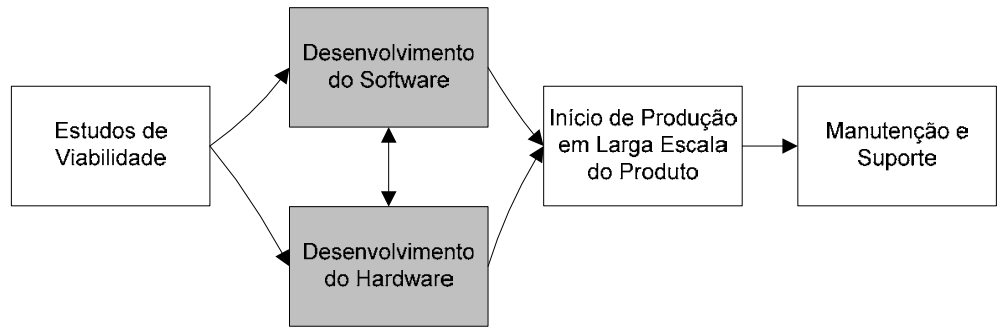

Figura 1. Ciclo de Vida dos Produtos de Software e Hardware da Maxtrack.

A partir da idéia e/ou necessidade de elaboração de um novo produto é realizado um estudo de viabilidade. Tal estudo visa a avaliar se os requisitos iniciais de hardware e de software desejados para o novo produto são factíveis com os componentes disponíveis e se é necessário a utilização de algum componente inovador. Feito isso, é iniciado o desenvolvimento do produto que, após ter sido completado, é enviado para produção em larga escala em uma das fábricas da empresa. Após o início da venda dos produtos, outra equipe, diferente da de desenvolvimento, fica responsável pela manutenção e pelo suporte aos usuários.

Até o início de 2005, a Maxtrack não seguia um processo de desenvolvimento formalizado. Devido a isto, a Alta Gerência da empresa decidiu iniciar um projeto de melhoria com a consultoria da COPPE/UFRJ. Na próxima seção será descrita a estratégia adotada pela COPPE/UFRJ para a atuação na Maxtrack.

\subsection{Estratégia da COPPE}

Para a definição do processo de desenvolvimento a ser utilizado pela Maxtrack, a consultoria da COPPE/UFRJ baseou-se na norma ISO/IEC 12207 (ISO/IEC, 2000) e nas práticas previstas para o CMMI Nível 2 (Chrissis et al., 2003). Além disso, boas práticas de Engenharia de Software, já utilizadas anteriormente com sucesso pela COPPE/UFRJ em outras oportunidades (Ferreira et al., 2005) (Duarte et al., 2005), também foram levadas em consideração. A cultura organizacional da Maxtrack foi respeitada na definição dos processos, influenciando aspectos como a condução de testes de software e a elaboração de modelos de análise e projeto.

Apesar de no Nível 2 do CMMI não ser necessária a definição de processos padrões para a organização, esta prática facilita a implantação dos processos além de já atender a práticas previstas no Nível 3 do CMMI (o que pode ser um facilitador quando a organização desejar no futuro uma avaliação Nível 3). Para a definição dos processos padrão e especializados (para Análise Estruturada e Orientada a Objetos) foi utilizada a abordagem descrita por Villela et al. (2004). Os processos de desenvolvimento em uso pela Maxtrack seguem uma estrutura padrão composta por 7 fases distintas, tendo, cada uma, um propósito particular: Planejamento Inicial do Projeto, Requisitos e Arquitetura, Planejamento do Projeto, Análise e Projeto, Construção e Testes, Integração do Sistema; e Implantação e Encerramento do Projeto.

Como apoio ferramental para a execução dos processos foi utilizada a Estação Taba (Villela et al., 2004)(Oliveira et al., 2004) que permite a configuração de ambientes de acordo com os processos e a cultura de uma organização. Atualmente, os ambientes Taba fornecem apoio às práticas dos Níveis 2 e 3 do CMMI (Rocha et al., 2005). 


\subsection{Etapas do Projeto}

Uma primeira tentativa de implantação de um programa de qualidade na Maxtrack foi realizada em 2004 e incluiu um ciclo de palestras com treinamento da COPPE/UFRJ cobrindo aspectos como Engenharia de Software, Engenharia de Requisitos, Gerência de Projetos, Gerência de Configuração, Inspeção e Testes, e Qualidade de Processos e Produtos de Software. Esta primeira tentativa foi interrompida logo após os treinamentos e recomeçou em 2005.

Nesta segunda fase do projeto, com o foco principal na melhoria da qualidade do desenvolvimento, e também objetivando a avaliação CMMI Nível 2, foram seguidas as seguintes etapas: (1) Definição da $1^{\mathrm{a}}$ versão do processo de desenvolvimento da Maxtrack (contemplando apenas a construção do Software) e configuração da Estação TABA para utilização deste processo; (2) Treinamentos em Engenharia de Software e no processo definido; (3) Definição do escopo da avaliação e nomeação dos grupos de processo; (4) Acompanhamento dos Projetos (Mentoring); (5) Definição da $2^{\mathrm{a}}$ versão do processo de desenvolvimento (dessa vez com uma evolução da versão para software e um novo processo para hardware e software); 6) Pré-avaliação CMMI (Readiness Assessment); (7) Ajustes em Relação aos Resultados do Readiness; (8) Avaliação Formal no CMMI Nível 2 (SCAMPI). Um cronograma dessas etapas é apresentado na Tabela 1.

Tabela 1. Cronograma de atividades realizadas.

\begin{tabular}{|c|c|c|c|c|c|c|c|}
\hline $\begin{array}{c}\text { Etapa } \\
\text { Mês }\end{array}$ & Jul/2005 & Ago/2005 & Set/2005 & Out/2005 & Nov/2005 & Dez/2005 & Jan/2006 \\
\hline $\mathbf{1}$ & $\mathrm{X}$ & & & & & & \\
\hline $\mathbf{2}$ & $\mathrm{X}$ & $\mathrm{X}$ & & & & & \\
\hline $\mathbf{3}$ & $\mathrm{X}$ & & & & & & $\mathrm{X}$ \\
\hline $\mathbf{4}$ & $\mathrm{X}$ & $\mathrm{X}$ & $\mathrm{X}$ & $\mathrm{X}$ & $\mathrm{X}$ & $\mathrm{X}$ \\
\hline $\mathbf{5}$ & & $\mathrm{X}$ & $\mathrm{X}$ & & & & \\
\hline $\mathbf{6}$ & & & & & & $\mathrm{X}$ & \\
\hline $\mathbf{7}$ & & & & & & $\mathrm{X}$ & $\mathrm{X}$ \\
\hline $\mathbf{8}$ & & & & & & & $\mathrm{X}$ \\
\hline
\end{tabular}

A definição dos processos (etapas 1 e 5) é descrita na seção 2.4. Os treinamentos (etapa 2) foram ministrados em forma de palestras por consultores da COPPE/UFRJ. A etapa 3 é descrita na seção $2.3 \mathrm{O}$ treinamento nas ferramentas da Estação TABA foi realizado durante o acompanhamento das atividades nos projetos (etapa 4). As etapas 6 , 7 e 8 são descritas na seção 2.5 .

\subsection{Etapa Inicial}

Como etapa inicial da implantação do programa de qualidade foi necessária a condução de três atividades: escolha dos projetos piloto, definição do escopo da avaliação CMMI, e definição dos grupos de processo da organização.

A escolha dos quatro projetos piloto levou em consideração a previsão de término próxima da avaliação SCAMPI e a alocação de um número de profissionais que fosse representativo do tamanho da organização. Os projetos escolhidos contemplavam o desenvolvimento de hardware e software. As equipes dos projetos foram constituídas por 1 Gerente de Projeto e 1 a 2 desenvolvedores (responsáveis por atividades de Análise, Projeto, Codificação e Planejamento dos Testes), 1 testador e 1 documentador. 
Como parte do desenvolvimento de produtos na Maxtrack é dependente da compra de circuitos eletrônicos e da elaboração de placas e caixas protótipo foi incluída a área de processo de Gerência de Acordo com Fornecedores (Supplier Agreement Management) no escopo da avaliação. Compras de software de prateleira (como editores de texto e ferramentas para gerência de projetos) não ficaram sobre o escopo desta área de processo, sendo tratadas à parte. Além disso, como o produto desenvolvido pela Maxtrack inclui a construção de hardware, decidiu-se pela avaliação SCAMPI segundo a disciplina Systems Engineering.

A execução dos processos não acontece exclusivamente durante a execução de projetos de software, ocorrendo também no âmbito organizacional, envolvendo o trabalho de três diferentes grupos: Gerência de Configuração, Métricas, e Garantia da Qualidade do Processo e do Produto (GQPP). A estrutura dos grupos e seus relacionamentos com os projetos são ilustrados na Figura 2.

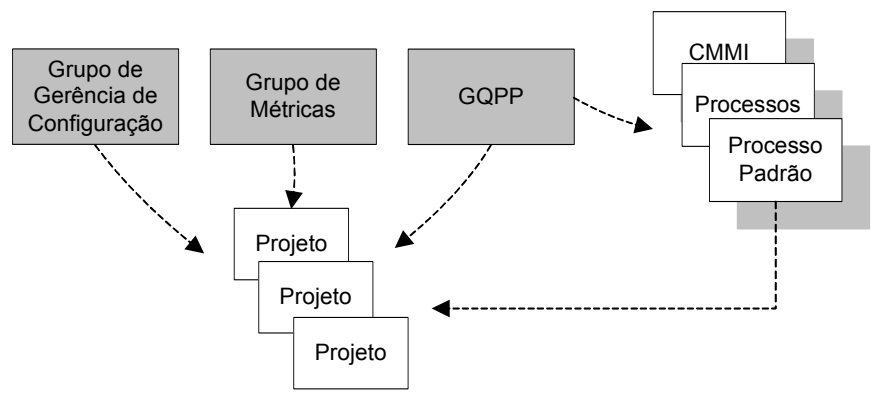

Figura 2. Estrutura dos Grupos de Processos

O GQPP atua como um auditor interno, ao avaliar o trabalho realizado pelos demais membros da organização. De forma semelhante, deve-se ter um mecanismo estabelecido na organização que garanta que o GQPP também esteja realizando suas tarefas adequadamente. Esta verificação encontra-se a cargo de uma auditoria externa realizada pela empresa Trails.

Foram escolhidos dois colaboradores da Maxtrack para serem membros do GQPP. Ambos fazem parte da equipe do suporte e documentação da empresa e, como tal, entendem perfeitamente os problemas que podem decorrer de um desenvolvimento com pouca qualidade. Para o Grupo de Métricas foi selecionado um profissional encarregado da realização dos testes dos produtos. Para o Grupo de Gerência de Configuração foram escolhidos dois dos três gerentes de projetos.

\subsection{Definição e Evolução dos Processos de Desenvolvimento da Maxtrack}

A execução dos projetos piloto permitiu a identificação de oportunidades de melhoria identificadas pelos participantes dos projetos (gerentes, desenvolvedores e membros do GQPP) além das demais pessoas envolvidas no âmbito organizacional (Grupo de Métricas e de Gerência de Configuração). Com a execução dos projetos piloto também se verificou a necessidade de um processo específico para quando o produto a ser desenvolvido contivesse itens de software e hardware. Todos os participantes dos projetos foram encorajados a elaborarem solicitações de melhoria nos processos da empresa. Dessa forma, o processo de desenvolvimento inicial implantado na Maxtrack sofreu uma revisão ao longo do projeto e deu origem a dois outros processos: a versão 2 do Processo para Desenvolvimento de Software e a versão 1 do Processo para 
Desenvolvimento de Hardware e Software.

As principais diferenças entre a versão para software e para hardware e software dos processos da Maxtrack são ilustradas na Fig. 3. As maiores diferenças estão: na Fase 2, com o a inclusão de uma atividade para Análise de Requisitos de Hardware; na Fase 4, com a inclusão de uma atividade para elaboração do Modelo de Análise\&Projeto do Hardware; na Fase 5, com a inclusão de uma atividade para Construção do Hardware (o que inclui montagem, avaliação e produção de protótipo para placa de circuito impresso) e a exclusão da atividade de homologação externa (pois os produtos de hardware não são produzidos para um cliente específico, se comportando quase como um produto de prateleira); na Fase 6 que passa a ser obrigatória e envolve a integração entre o software e o hardware produzidos além da integração com outros sistemas; e na Fase 7 com a exclusão das atividades referentes a documentação do usuário e implantação do produto.
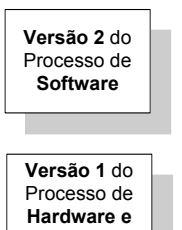
Software
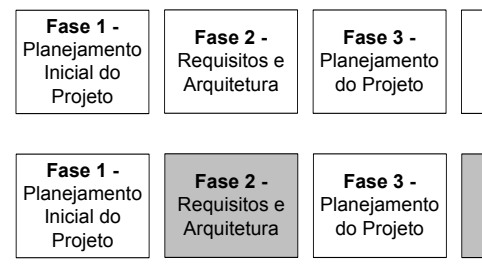
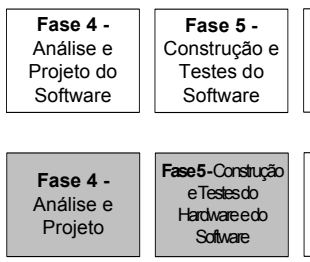
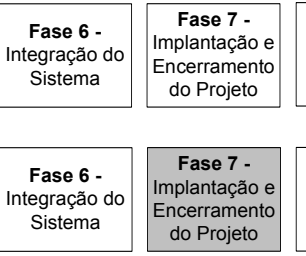

Atividades Comuns às Fases

Atividades
Comuns às
Fases
Fases

Figura 3. Evolução do Processo de Software e Hardware da Maxtrack

Um dos projetos piloto, de desenvolvimento de hardware e software, continuou utilizando a versão inicial enquanto outros dois, cujo desenvolvimento era de hardware e software, foram migrados para a nova versão do processo e as atividades que não eram ainda previstas no processo original foram realizadas. O quarto projeto era de desenvolvimento apenas de um produto de software e foi migrado para a segunda versão do processo, porém foi descontinuado por motivos técnicos. Um novo projeto foi iniciado já utilizando a versão do processo de hardware e software.

\subsection{A Avaliação SCAMPI}

A preparação para a avaliação SCAMPI começou no final de novembro de 2006 com a elaboração das planilhas de evidências a serem apresentadas à equipe de avaliação responsável pelo Readiness. Nesta pré-avaliação, a empresa deve submeter à apreciação evidências que comprovem que os processos estão aderentes às práticas do CMMI e que estes processos foram, estão sendo e continuarão a serem seguidos pela empresa. Por decisão da empresa, todos os documentos dos processos só existem em meio eletrônico, salvo raras exceções, como alguns documentos relativos a Gerência de Acordo com Fornecedores, como recibos e contratos.

Durante o Readiness, foi pedido que os membros do GQPP apresentassem os processos de desenvolvimento em uso na empresa e explicassem os mecanismos utilizados pela empresa para seguir os processos. Após a análise das evidências, ao final do Readiness, potenciais pontos fracos, pontos fortes e oportunidades de melhoria foram identificados pela equipe de avaliação. Para cada ponto fraco e oportunidade de melhoria identificados foi elaborado um plano de ação a ser executado até a avaliação SCAMPI.

Os projetos avaliados continuaram a ser executados e as evidências (tanto as já apresentadas à equipe de avaliação e outras sugeridas por ela) foram atualizadas nas 
planilhas de evidências.

Durante a avaliação SCAMPI, ocorrida em janeiro de 2006, a planilha de evidências foi revista pela equipe de avaliação, as sessões de demonstração de ferramentas foram realizadas, e as entrevistas foram conduzidas. No total 7 pessoas foram entrevistadas: 1 gerente de métricas, 2 desenvolvedores, 2 membros do GQPP (que acumulavam a tarefa de execução de testes e documentação nos projetos) e 2 gerentes de projeto (ambos acumulavam a função de gerente de configuração e 1 deles era responsável também pelos acordos com os fornecedores). Apenas um dos desenvolvedores dos projetos avaliados não foi entrevistado, pois estava participando de um projeto no Chile.

Como resultado da avaliação SCAMPI, a equipe de avaliação apresentou os pontos fortes da empresa e as fraquezas (que não foram consideradas substanciais) encontradas e a empresa conseguiu alcançou o Nível 2 do CMMI como pretendido.

\section{Resultados e Lições Aprendidas}

O maior resultado, para a Maxtrack, da implantação de seu programa de qualidade foi a melhoria no desenvolvimento de seus produtos e a obtenção do Nível 2 do CMMI apenas evidencia este fato. Com essa experiência a empresa mostra que uma organização pode ser avaliada mesmo com uma equipe reduzida e um curto espaço de tempo de preparação. Com essa experiência foi possível identificar alguns fatores de sucesso para a Maxtrack: ter incluído nos processos organizacionais boas práticas de engenharia de software; ter os processos padrões definidos e institucionalizados desde o Nível 2; manter o rigor na implantação dos processos desde o início dos projetos; contar com o apoio de uma consultoria especializada; ser auditada por uma entidade externa de forma regular e eficiente; investir no treinamento de pessoal; ter na empresa uma equipe de qualidade que seja capaz de avaliar o conteúdo dos documentos produzidos; ter apoio de ferramentas adequadas, como a Estação TABA (cuja utilização foi apontada como ponto forte no apoio a algumas práticas do modelo), para auxiliar a implantação dos processos.

Apenas ter bons processos e executá-los correta e adequadamente não é suficiente para se obter uma avaliação CMMI, é preciso evidenciar para uma equipe de avaliação todos os procedimentos e boas práticas executadas de acordo com as práticas do modelo. Dessa forma, durante a preparação para a pré-avaliação é importante: (i) minimizar o número de documentos a serem analisados pela equipe de avaliação, escolhendo os documentos mais significativos para evidenciar o cumprimento da prática e, se a prática tem intersecção com outra, escolher um documento que sirva a ambas; (ii) manter os documentos apenas em meio eletrônico também facilita a análise da equipe de avaliação; (iii) manter, durante a pré-avaliação, pessoas chave para o programa de qualidade disponíveis para auxiliar a equipe de avaliação a entender os documentos gerados pela empresa, pois o mau entendimento de uma evidência pode fazer com que seja, indevidamente, considerada uma fraqueza.

Além disso, durante a avaliação é importante manter uma equipe disponível para conversar com os entrevistados antes das entrevistas para tirar as dúvidas sobre o SCAMPI e deixá-los cientes e confiantes de que nenhum tipo de represália será efetuada, uma vez que há um termo de confidencialidade para a realização da avaliação. 


\section{Considerações Finais}

Este artigo apresentou a experiência da Maxtrack na implantação de um programa de qualidade no desenvolvimento de software. A implantação desse programa culminou na obtenção do Nível 2 do CMMI. Como próximos passos da empresa estão a finalização dos projetos em andamento (nenhum deles estava finalizado durante a avaliação SCAMPI) e o início de outros projetos ainda seguindo os processos atuais (compatíveis com o Nível 2 do CMMI). Além disso, uma revisão do processo de desenvolvimento será realizada de forma a garantir uma maior integração das práticas de Gerência de Acordos com Fornecedores e um maior controle de gerência de configuração sobre os produtos de hardware. Para o segundo semestre de 2006 está previsto o início da definição e implantação de novas versões dos processos visando à obtenção do Nível 3 do CMMI.

\section{Agradecimentos}

Os autores agradecem a todos os colaboradores da Maxtrack que com seu empenho e entusiasmo tornaram possível a conquista do CMMI Nível 2 e a Silvia Vaisburn da Trails pela eficiência e contribuições como avaliador externo. Agradecem, também, a toda a equipe de avaliação SCAMPI e à Viviana Rubinstein da Liveware, que liderou a avaliação, pela seriedade no trabalho e sugestões de melhoria. Finalmente agradecem à equipe de desenvolvimento da Estação TABA e a Guilherme Travassos pelo suporte ao longo de todo o projeto.

\section{Referências}

Chrissis, M. B., Konrad, M, Shrum, S. "CMMI: Guidelines for Process Integration and Product Improvement”, Addison-Wesley, 2003.

Duarte, E., Silva R., Natali, A., Rocha, A., Santos, G. (2005) "Uma Abordagem para Implantação de Processos de Software com ISO 9001 e CMMI", IV Simpósio Brasileiro de Qualidade de Software, SBQS'05, Porto Alegre, RS, Brasil.

Ferreira, A., Cerqueira, R., Rocha, A., Santos, G., Montoni, M., Mafra, S., Figueiredo, S. (2005) "Implantação de Processo de Software na BL Informática Um Caso de Sucesso", IV Simpósio Brasileiro de Qualidade de Software, SBQS'05, Porto Alegre, RS, Brasil.

ISO/IEC 12207:2000 - Information technology - software process life cycle, (2000).

ISO 14001:2004 - Environmental management systems, (2004)

ISO 9001:2000 - Quality management systems - Requirements, (2000)

Oliveira, K, Zlot, F., Rocha, A. R., Travassos, G., Galotta, C., Menezes, C. Domain Oriented Software Development Environment, Journal of Systems and Software, vol 72/2 (2004) pp 145-161

Rocha, A. R., Montoni, M., Santos, G., Mafra, S., Figueiredo, S., Bessa, A., Mian, P. (2005) "Estação TABA: Uma Infra-estrutura para Implantação do Modelo de Referência para Melhoria de Processo de Software", IV SBQS, Porto Alegre, Brasil.

Villela, K., Santos, G., Montoni, M., Berger, P., Figueiredo, S, M., Mafra, S, N., Rocha, A, R, C., Travassos, G, H. (2004) "Definição de Processos em Ambientes de Desenvolvimento de Software Orientados a Organização", III Simpósio Brasileiro de Qualidade de Software, Brasília-DF. 